\title{
Large Area Polycrystalline Diamond Detectors for Online Hadron Therapy Beam Tagging Applications
}

\author{
ML. Gallin-Martel Member IEEE, A. Bes, A. Boukhémiri, G. Bosson, J. Collot, D. Dauvergne Member IEEE, M. \\ Fontana, L. Gallin-Martel, A. Gorecki, JY. Hostachy, J. Krimmer, A. Lacoste, S. Marcatili, J. Morse, JF. Muraz, FE. \\ Rarbi, O. Rossetto, M. Salomé, E. Testa, M. Yamouni
}

\begin{abstract}
The French national collaboration CLaRyS aims at the on-line monitoring of hadron therapy by means of imaging nuclear reaction products that are related to the ion range. For this purpose, the MoniDiam project is dedicated to the development of a large area detector with a high detection efficiency for the primary carbon or proton beams, giving time and position measurements at count rates greater than $100 \mathrm{MHz}$. High radiation hardness and intrinsic electronic properties make diamonds reliable and very fast detectors with a good signal to noise ratio. Commercial Chemical Vapor Deposited (CVD) diamonds are studied starting with plasma etch thinning and contact metallization done at the LPSC laboratory. Their applicability as particle detector is being investigated using $\alpha$ and $\beta$ radioactive sources, $95 \mathrm{MeV} / \mathrm{u}$ carbon ion beams and shortbunched $8.5 \mathrm{keV}$ photons from the European Synchrotron Radiation Facility (ESRF). This last facility offers unique capability of highly focused beams, the $\mathrm{X}$-ray energy deposition is continuous along the irradiated detector volume. It permits us to mimic the interaction of single ion beams. The possible application of diamonds as a position sensitive detector will be discussed from these radiation tests results. Finally, we focused on the diamond time response and energy resolution measurements. The reported study prefigures dedicated microelectronics developments.
\end{abstract}

\section{INTRODUCTION}

$\mathrm{T}$ HE treatment of tumors by a beam of carbon ions or protons is an alternative cancer therapy known as hadron therapy. The ions deposit a large fraction of their dose at the end of their path, in the Bragg peak. Compared to conventional Xray radiotherapy, it allows a more efficient dose delivery in the tumor, with a reduction of the dose deposited in the nearby healthy organs. One of the main issues is the conformation of the dose to the tumor volume, provided by multiple Bragg peak juxtaposition. Since multiple sources of uncertainty on the ion range may cause deviations from the planned dose distribution [1], online control of the ion range is required.

During an irradiation with hadron beams, photons in the range $1-10 \mathrm{MeV}$ are emitted almost isotropically within much less than a picosecond after nuclear reactions occurred on the hadron path in the patient body. It has been shown that the longitudinal distribution of these prompt-gamma production is

Manuscript received November 29, 2016. This work was supported by Labex Primes, France Hadron and MONODIAM-HE.

ML. Gallin-Martel, A. Bes, A. Boukhémiri, G. Bosson, J. Collot, D. Dauvergne, L. Gallin-Martel, A. Gorecki, JY Hostachy, A. Lacoste, S. Marcatili, JF Muraz, FE. Rarbi, O. Rossetto and M. Yamouni are with Laboratoire de Physique Subatomique et de Cosmologie, CNRS/IN2P3, highly correlated to the primary ion range [2], [3]. In the CLaRyS collaboration, two systems for prompt gamma detection are currently under development [4], [5], [6]: a collimated gamma camera and a Compton camera (Fig. 1). The originality of the two present systems is the use of a beam tagging hodoscope. It plays a major role in all the detection concepts as it provides position and timing information of the incident hadron beam. This hodoscope relies on an array of scintillating fibres which are coupled to multichannel photomultiplier tubes (PMT). However, the performances of such a design are limited by the scintillating fibers and PMT timing resolutions. Their admissible counting rate and the intrinsic radiation hardness of the scintillators are also two important issues. To overcome such difficulties, the collaboration has initiated the MoniDiam project which aims at developing a diamond based hodoscope and its dedicated integrated fast read-out electronics.

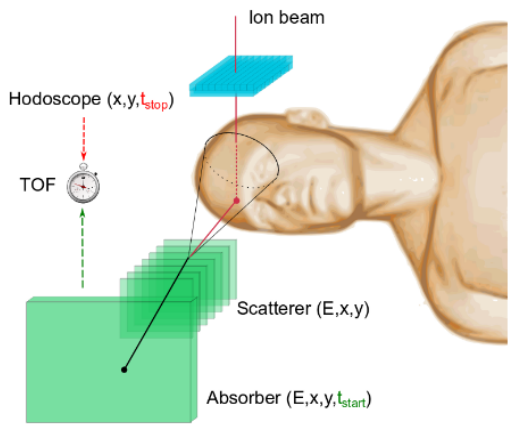

Fig. 1 Scheme of the combination of a Compton camera and a beam hodoscope for time of flight prompt-gamma range verification.

\section{DIAMOND SENSORS}

\section{A. Polycrystalline CVD Diamond}

Polycrystalline CVD diamond is a synthetic diamond material grown by the technique of microwave plasma

Université Grenoble-Alpes, UMR 582138026 Grenoble Cédex France (corresponding author ML. Gallin-Martel e-mail : mlgallin@lpsc.in2p3.fr)

M. Fontana, J. Krimmer and E. Testa are with IPNL, Université de Lyon, Université Lyon 1, CNRS/IN2P3, UMR 5822, France,

M. Salomé and J.Morse are with ESRF, Grenoble, France 
enhanced chemical vapor deposition. It can be grown to large areas $\left(2 \times 2 \mathrm{~cm}^{2}\right.$ are commercially available) and its qualities and detection properties are reproducible by tightly controlling its growth parameters. Its radiation hardness has already been demonstrated. Compared to other semiconductor detectors, such as silicon ones, detectors based on CVD diamonds exhibit several advantages. A high resistivity $\left(>10^{13} \Omega \mathrm{m}\right.$ ) coupled to a large electronic gap $(5.48 \mathrm{eV})$ results in a lower noise level and an almost negligible leakage current. Despite the fact that the energy required to produce electron-hole pairs is 3.6 times larger than in silicon, the signal to noise ratio is higher for diamond. The high charge carrier mobility leads to a very fast detector response allowing excellent time resolution (few tens of picoseconds) and high counting rates to be reached. Hence diamond applications are more and more considered in various physics fields. Diamond detectors are commonly used as X-ray synchrotron radiation monitors but also as UV and neutron sensors [7]. In high energy physics, diamond detectors are used for beam monitoring [8]. In medical physics applications they are used as dosimeters for radiotherapy [9].

\section{B. Metallization and electrical contact}

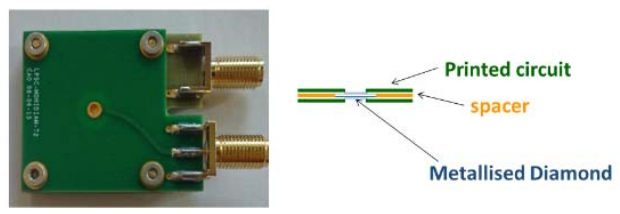

Fig. 2 The diamond sensor housed in its socket.

Poly- and single-crystal CVD diamond samples produced by different manufacturers were used. Their metallization was locally performed by using the DMW (Distributed Microwave Plasmas), a fully original technology developed by LPSC [10]. The crystal surface preparation and metal deposition were thus performed by a sequential plasma process consisting in two steps of reactive plasma processing followed by plasmaassisted sputtering. The sensor contact consists of a $50 \mathrm{~nm}$ thick aluminum layer deposited both on the growth and the substrate sides. This process allows strip metallization (one side in $\mathrm{X}$ direction and the other on $\mathrm{Y}$ direction) for a position sensitive detector development.

Diamond samples, with aluminum surface metallization, are mounted on sample holders with $50 \Omega$ adapted impedance and SMA connectors, enabling reversible bias and signal readout from both sides of the diamond as illustrated by Fig. 2 .

\section{CHARACTERIZATION OF CVD DIAMOND}

\section{A. Leakage current measurement.}

The quality of the contacts was inferred by measuring the current voltage characteristics. For this purpose, a dedicated test bench was developed. This test setup was also used to highlight possible diamond sparking. Table 1 summarizes the leakage current measurements on various diamond samples by varying the bias voltage. The measured currents are lower than $10 \mathrm{pA}$.
The charge collection properties of CVD diamond were then investigated with various ionizing particles. We aimed to evaluate the capability of diamond to be used as position sensitive detector. The tested samples were ranging from 300 $\mu \mathrm{m}$ up to $500 \mu \mathrm{m}$ in thickness.

TABLE 1 LEAKAGE CURRENT MEASUREMENT (UNITS ARE PICO AMPS) ON VARIOUS DIAMOND SAMPLES AS A FUNCTION OF BIAS VOLTAGE (SC STANDS FOR SINGLE CRYSTAL AND PC STANDS FOR POLY CRYSTAL)

\begin{tabular}{|c|c|c|c|c|c|c|}
\hline \multirow[t]{2}{*}{ Diamond } & \multicolumn{6}{|c|}{$\begin{array}{l}\text { HT } \\
(\mathrm{V})\end{array}$} \\
\hline & 0 & 100 & 200 & 300 & 400 & 500 \\
\hline $\begin{array}{c}\text { sc-CVD } \\
0.45 \times 0.45 \mathrm{~cm}^{2} \times 518 \mu \mathrm{m}\end{array}$ & 3.45 & 2.90 & 2.59 & 2.20 & 2.29 & 2.98 \\
\hline $\begin{array}{c}\text { pc-CVD } \\
1 \times 1 \mathrm{~cm}^{2} \times 500 \mu \mathrm{m}\end{array}$ & 0.99 & 2.72 & 4.32 & 6.21 & 7.16 & 9.58 \\
\hline $\begin{array}{c}\text { pc-CVD } \\
2 \times 2 \mathrm{~cm}^{2} \times 500 \mu \mathrm{m} \\
\end{array}$ & 0.4 & 1.28 & 2.21 & 3.77 & 6.23 & 10.9 \\
\hline
\end{tabular}

B. Measurements with alpha particles.

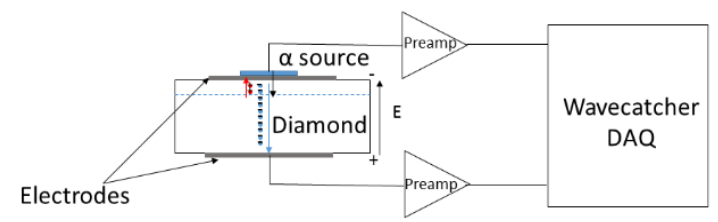

Fig. 3 Scheme of the charge collection measurement

At first, tests were carried out using ${ }^{233} \mathrm{U}$ and ${ }^{241} \mathrm{Am}$ sources (Fig. 3). Since the mean free path of the $\alpha$ particles (about 12 $\mu \mathrm{m}$ ) is much smaller than the diamond thickness, all the current due to the migration of charge carriers comes from a single species (either electrons or holes) traversing the whole detector thickness. This enabled us to explore the diamond sensitivity to polarization effects. The response signal was also used to extract information about the difference on the growth and substrate sides.

\section{Measurements with a pulsed photon beam $(8.5 \mathrm{keV})$.}

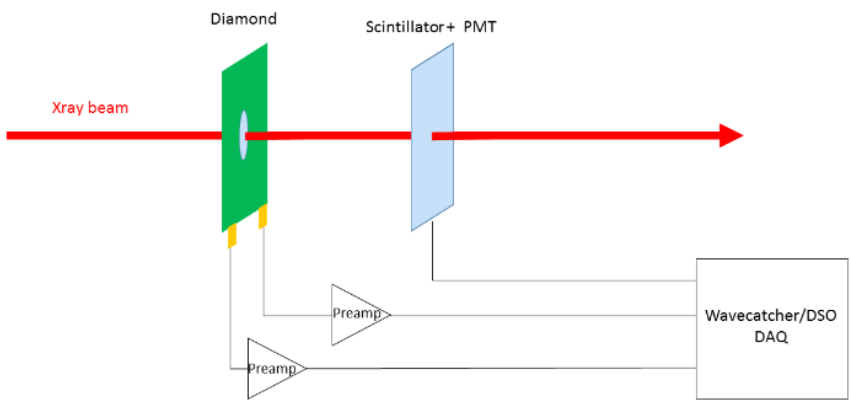

Fig. 4 Experimental set-up at the ESRF facility.

A $8.5 \mathrm{keV}$ photon focused micro-beam with a well-defined time structure was used at the European Synchrotron Radiation Facility (ESRF) in Grenoble. As regards energy deposition in the diamond, in the ESRF 4-bunch mode, the $\sim 100$ ps duration $\mathrm{X}$-ray pulses, containing a fixed number of photons varying up to $\sim 1400$, spaced at $704 \mathrm{~ns}$ intervals, mimic the passage of single ionizing particles. Indeed, $35 \%$ of the beam is absorbed in $300 \mu \mathrm{m}$ diamond material, which makes the energy deposit 
almost constant along the beam path. We characterized the temporal and charge/current signal responses of several prototypes diamond detector over a large fraction of their surfaces with:

- sub-nanosecond timing using wide bandwidth acquisition of pulse waveforms,

- micron spatial resolution maps of the detectors using current integration mode with pico-ammeters.

The diamond samples were placed inside an electromagnetic shielding box. By means of a magnetic kinematic mount, the box was positioned with micrometric reproducibility at the sample position of the micro-diffraction end station (in air) of the ID21 beamline at ESRF. Fig. 4 illustrates this sample setup. Additionally, a plastic scintillator was placed downstream. It was readout by a photomultiplier tube (PMT). This was used for timing verification and detection efficiency comparison.

The pulse shape readout mode was performed alternatively with a $500 \mathrm{MHz}, 3.2 \mathrm{GS} / \mathrm{s}$ digital sampling 'Wavecatcher' [11] system, and with a $2 \mathrm{GHz}, 20 \mathrm{GS} / \mathrm{s}$ analog bandwidth DSO (LeCroy 620Zi) [12]. This double configuration was useful since the Wavecatcher system could be configured in a continuous acquisition mode, recording all waveforms, and thus enabling large statistics offline analysis.

The acquisition of the DSO was fully integrated into the ID21 SPEC software acquisition framework which enabled us to measure the temporal response of the detectors as they were motor scanned in the beam, thus creating 3D maps ( $\mathrm{x}, \mathrm{y}$ position and response time). Both acquisition systems were triggersynchronized by the RF of the ESRF synchrotron. The 100 msec current integration readout mode was performed by means of a Keithley 485 electrometer coupled to a voltage-tofrequency converter and a standard ESRF counter board.

Several preamplifiers were tested: the CIVIDEC C2 low noise broadband RF amplifier ( $2 \mathrm{GHz}, 40 \mathrm{~dB}$ from CIVIDEC Instrumentation company [13]); DBA III (Diamond Broadband Amplifier [14]) from GSI (a similar $2 \mathrm{GHz}$ design with a gain remotely controlled from $0 \mathrm{~dB}$ to $38 \mathrm{~dB}$ ) and a low cost current preamplifier designed at LPSC $(1 \mathrm{GHz}, 30 \mathrm{~dB}$ still under design). These amplifiers are intended for use with fast detectors. They exhibit a $50 \mathrm{ohms}$ input impedance and are able to work with FWHM pulse widths of less than 1 ns. Four synthetic CVD grown diamond detectors were tested: one single crystal and two polycrystalline from the manufacturer Element 6, and one diamond heteroepitaxially grown at the Augsburg University [15]. For the heteroepitaxial growth, a substrate with a diamond like structure is used as seed, in present case it is iridium.

\section{Measurements with a $95 \mathrm{MeV} / \mathrm{u}^{12} \mathrm{C}$ carbon beam.}

Finally, to evaluate the diamond performance under carbon ion radiations a test with a $95 \mathrm{MeV} / \mathrm{u}{ }^{12} \mathrm{C}$ carbon beam at the GANIL facility was carried out. The energy deposit by such ions in a $300 \mu \mathrm{m}$ thick diamond is $25 \mathrm{MeV}$. That time, two diamond samples were arranged one behind the other and a plastic scintillator read by a PMT was placed downstream. Furthermore, to focus the beam on the sensitive surface of the two diamond sensors a copper collimator was placed upstream (Fig. 5).

This experimental set-up permits us to evaluate single carbon ion detection efficiency of each tested diamond sample by comparing the measured rate of the double coincidences to the triple ones. The pulse shape readout mode was performed with the $500 \mathrm{MHz}, 3.2 \mathrm{GS} / \mathrm{s}$ digital sampling 'Wavecatcher' system.

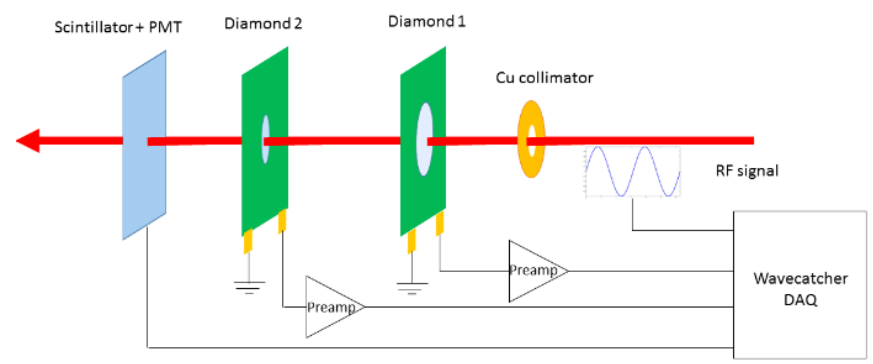

Fig. 5 Experimental set-up at the GANIL facility.

\section{RESUltS}

A. Time resolution measurements

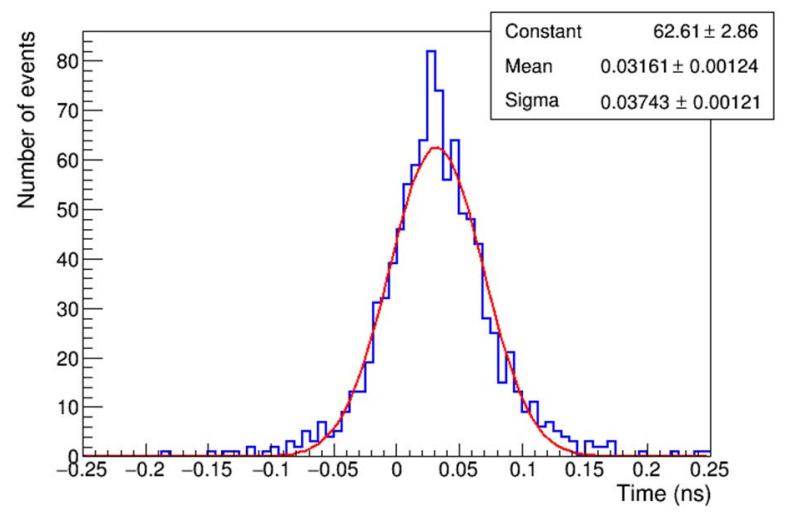

Fig. 6 Time resolution measured on a $2 \times 2 \mathrm{~cm}^{2}$ polycrystalline diamond (difference of the timing of both surface signals) measured with an $\alpha$ source.

An offline procedure was established to determine the time resolution of the detectors. The averaged readout signal waveforms are recorded independently on both faces of a detector. A numerical Constant Fraction Discrimination (CFD) was used by averaging the background, determining the maximum of the pulses, and interpolating the $50 \%$ rise time value. The distribution of the time difference between two faces of a detector is characteristic of the readout chain jitter. Fig. 6 represents such a distribution for a $500 \mu \mathrm{m}$ thick, $20 \times 20 \mathrm{~mm}^{2}$ polycrystalline detector under $\alpha$ particle radiation. In the present case the resolution is $37 \mathrm{ps}$ rms (to be divided by 1.414 for a single signal since this is an auto-convolution).

The summary of time resolution measurements for several detectors and several preamplifiers is given in Table 2, the referred measurements were done at the ESRF facility under a $8.5 \mathrm{keV}$ photon beam.

At the GANIL facility, the dedicated experimental set-up permits us to measure a time resolution between two diamond samples positioned one behind the other. Here a time resolution 
lower than 20 ps rms was reached using a $0.45 \times 0.45 \mathrm{~cm}^{2} \times 518$ $\mu \mathrm{m}$ single-crystal sample provided by Element 6 coupled to a $0.5 \times 0.5 \mathrm{~cm}^{2} \times 300 \mu \mathrm{m}$ poly-crystal grown heteroepitaxially provided by the Augsburg University.

Such results are very promising but rely on developments of very fast front-end electronics for our future prototype detector.

TABLE 2 TIME RESOLUTION MEASURED ON VARIOUS DIAMOND SAMPLES COUPLED TO VARIOUS PREAMPLIFIERS WITH A PULSED PHOTON BEAM (SC STANDS FOR SINGLE CRISTAL AND PC FOR POLY CRISTAL)

\begin{tabular}{|c|c|c|c|c|}
\hline & Diamond & Preamp. & $\begin{array}{l}\text { HT } \\
\text { (V) }\end{array}$ & $\begin{array}{c}\text { Time } \\
\text { Resolution } \\
\text { RMS } \\
\text { (ps). }\end{array}$ \\
\hline sc & $0.45 \times 0.45 \mathrm{~cm}^{2} \times 518 \mu \mathrm{m}$ & CIVIDEC & -500 & 26,7 \\
\hline sc & $0.45 \times 0.45 \mathrm{~cm}^{2} \times 518 \mu \mathrm{m}$ & CIVIDEC & 500 & 25,1 \\
\hline sc & $0.45 \times 0.45 \mathrm{~cm}^{2} \times 518 \mu \mathrm{m}$ & DBAIII & -500 & 48,84 \\
\hline sc & $0.45 \times 0.45 \mathrm{~cm}^{2} \times 518 \mu \mathrm{m}$ & DBAIII & 500 & 50,11 \\
\hline sc & $0.45 \times 0.45 \mathrm{~cm}^{2} \times 518 \mu \mathrm{m}$ & LPSC & -500 & 53,8 \\
\hline sc & $0.45 \times 0.45 \mathrm{~cm}^{2} \times 518 \mu \mathrm{m}$ & LPSC & 500 & 48,41 \\
\hline pc & $0.5 \times 0.5 \mathrm{~cm}^{2} \times 300 \mu \mathrm{m}$ & CIVIDEC & 300 & 49,22 \\
\hline pc & $1 \times 1 \mathrm{~cm}^{2} \times 500 \mu \mathrm{m}$ & CIVIDEC & 300 & 71,94 \\
\hline
\end{tabular}

B. Energy resolution measurement

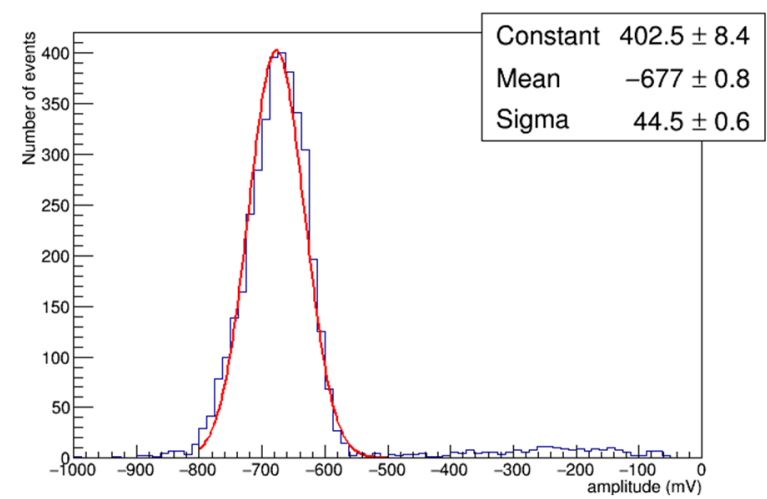

Fig. 7 Energy resolution measured on a $0.5 \times 0.5 \mathrm{~cm}^{2} \times 300 \mu \mathrm{m}$ polycrystalline diamond at the GANIL facility with $95 \mathrm{MeV} / \mathrm{u}$ carbon beam.

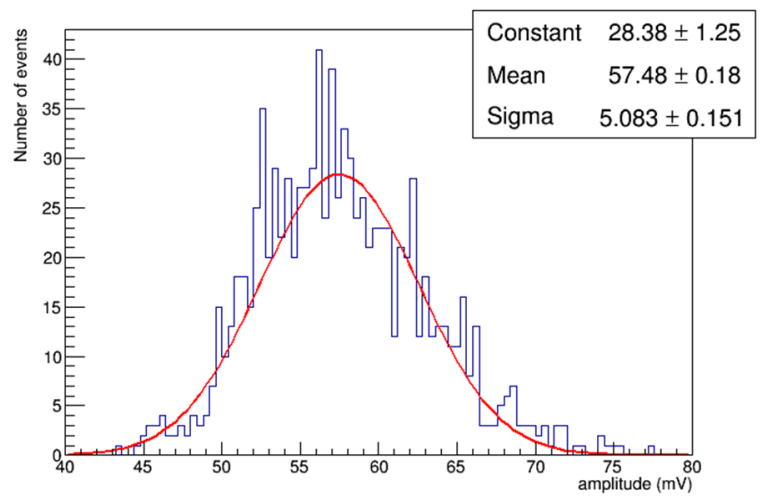

Fig. 8 Energy resolution measured on a $0.5 \times 0.5 \mathrm{~cm}^{2} \times 300 \mu \mathrm{m}$ polycrystalline diamond at the ESRF facility with a pulsed photon beam $(8.5$ $\mathrm{keV})$.
Energy resolution measurements were performed both at the ESRF and GANIL facilities. With a $95 \mathrm{MeV} / \mathrm{u}$ carbon ion beam at the GANIL facility impacting a $0.5 \times 0.5 \mathrm{~cm}^{2} \times 300 \mu \mathrm{m}$ polycrystalline diamond, an energy resolution at the level of 7 $\%$ rms was reached (Fig. 7). Using the same diamond sensor, with the $8.5 \mathrm{keV}$ pulsed photon beam at ESRF an energy resolution at the level of $9 \% \mathrm{rms}$ was obtained (Fig. 8).

\section{Surface mapping.}
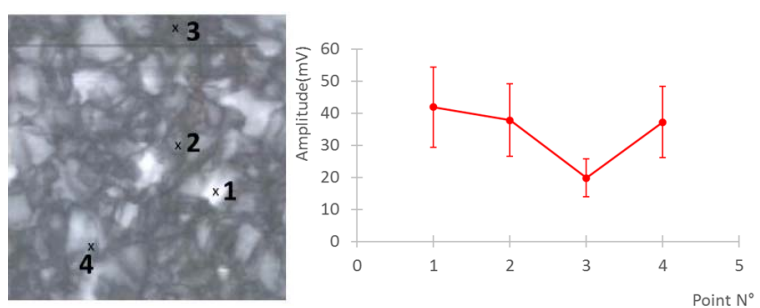

Fig. 9 Amplitude (right) of the signal versus the pencil beam localization from point 1 up to 4 on the surface map (left) of a $1 \mathrm{~mm}^{2}$ area of a $1 \times 1 \mathrm{~cm}^{2}$ x $500 \mu \mathrm{m}$ polycrystalline diamond detector

Surface mapping of several detectors was performed. Fig. 9 shows an example of a polycrystalline detector map $\left(1 \times 1 \mathrm{~cm}^{2}\right.$ $\times 500 \mu \mathrm{m}$ detector from Element 6 ). The grey scale corresponds to the charge collection efficiency measured by an electrometer. Clearly, the response of the detector reflects the spatial distribution of grain boundaries in the polycrystalline material. The case presented here corresponds to the worst variations measured, chosen as the image shows good contrasts.

In addition, Fig. 9 shows the variation of the amplitude of the signal versus the pencil photon beam localization for the same pc-CVD diamond detector. With such a sample, a factor two of difference is observed between the clearest point and the darkest one.

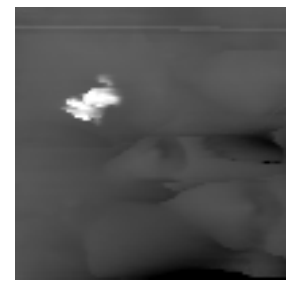

Fig. 10 Surface map of a $1 \mathrm{~mm}^{2}$ area of the $0.5 \times 0.5 \mathrm{~cm}^{2} \times 300 \mu \mathrm{m}$ heteroepitaxially grown diamond on Iridium sample from Augsburg University.

On the contrary, the heteroepitaxially grown diamond on Iridium sample from the Augsburg University laboratory exhibited a much more homogeneous response (Fig. 10) which that time confirms the nice measured energy resolution observed on both Fig. 7 and Fig. 8. Such a sample is well suited for our beam monitoring application. The clearest area on the left top corner in Fig. 10 stands for a very located defect in the crystal structure which has been found to have poor influence on the overall measurements. 


\section{CONCLUSION}

Synthetic pc-CVD diamond detectors are foreseen for online hadron therapy beam tagging applications. They will be used as a hodoscope which plays a major role for particle identification both in a gamma camera and Compton camera projects proposed by the CLaRyS French collaboration. Their radiation hardness, fast response and good signal to noise ratio make diamonds good candidates. A time resolution ranging from 20 up to $40 \mathrm{ps}$ rms and an energy resolution ranging from 7 up to $9 \%$ rms were measured irradiating the whole surface of pc-CVD diamond using various ionizing particles despite the obvious non uniformity of the crystalline structure observed thanks to X-ray analyses.

The final foreseen detector would consist in a $\sim 15 \times 15 \mathrm{~cm}^{2}$ mosaic arrangement of stripped sensors read by a dedicated integrated electronics ( $\sim 1800$ channels). The expected counting rate per channel is $10 \mathrm{MHz}$, the time resolution is at the level of few tens of picoseconds and the spatial resolution is at the level of $1 \mathrm{~mm}$ (readout strip width).

\section{ACKNOWLEDGMENT}

The authors would like to acknowledge the ESRF for provision of synchrotron radiation facilities and would like to thank the ID21 beamline staff for their assistance with experiment MI-1243. This work was supported by the Labex PRIMES (ANR-11-LABX-0063), FranceHadron (ANR-11INBS-0007) and ANR MONODIAM-HE (ANR-089520). The authors are grateful to Matthias Schreck from the Augsburg University for providing the LPSC laboratory with samples of diamond heteroepitaxially grown. Dominique Breton from the Laboratoire de l'Accélérateur Linéaire and Eric Delagnes from CEA Saclay are thanked for their implication in dedicated software development and technical support of the namely "wavecatcher" data acquisition system.

\section{REFERENCES}

[1] H. Paganetti, "Range uncertainties in proton therapy and the role of Monte Carlo simulations," Phys. Med. Biol., vol. 57, no. 11, pp. R99-R117, Jun. 2012.

[2] C.-H. Min, C. H. Kim, M.-Y. Youn, and J.-W. Kim, "Prompt gamma measurements for locating the dose falloff region in the proton therapy," Appl. Phys. Lett., vol. 89, no. 18, pp. 183517-3, Oct. 2006.

[3] E. Testa et al., "Monitoring the Bragg peak location of $73 \mathrm{MeV} / \mathrm{u}$ carbon ions by means of prompt $\gamma$-ray measurements," Appl. Phys. Lett., vol. 93, no. 9, p. 93506, 2008.

[4] J. Krimmer et al., "Collimated prompt gamma TOF measurements with multi-slit multi-detector configurations," J. Instrum., vol. 10, no. 1, pp. P01011-P01011, Jan. 2015.

[5] J. Krimmer et al., "Development of a Compton camera for medical applications based on silicon strip and scintillation detectors," Nucl. Instrum. Methods Phys. Res. Sect. Accel. Spectrometers Detect. Assoc. Equip, Elsevier, 787, pp 98-101,2015 < doi:10.1016/j.nima.2014.11.042>.

[6] J. Krimmer et al., "Real-Time Online Monitoring of the Ion Range by Means of Prompt Secondary Radiations", published in 3rd International Conference On Advancements In Nuclear Instrumentation Measurement Methods And Their Applications (ANIMMA 2013), Marseille: France 2013.

[7] C. Weiss et al., n-TOF collaboration, "A new CVD diamond mosaicdetector ofr $(n, \alpha)$ cross-section measurements at the n-TOF experiment at CERN", proceedings, $13^{\text {th }}$ Vienna Conference on Instrumentation (VCI
2013), Vienna, Austria, February 11-15, 2013, Nucl. Instrum.Meth A732 pp 190-194, 2013.

[8] E. Griesmayer and B. Dehning, "Diamonds for beam instrumentation", in Proceedings of the $2^{\text {nd }}$ International Conference on Technology and Instrumentation in Particle Physics (TIPP 2011), Chicago U.S.A. (2011) [Phys. Procedia 37 p 1997, 2012].

[9] http://www.ptw.de/2968.html

[10] A. Lacoste et al., "Multi-dipolar plasmas for uniform processing: physics", design and performance, SCi. Technol., 11 pp 407-412, 2002.

[11] D.Breton, E. Delagne, J. Maalmi, "Picosecond time measurement using ultra-fast analog memories", proceedings TWEPP2009, Topical Workshop on Electronics for Particle Physics. Paris 21-25 September 2009

[12] http://teledynelecroy.com/oscilloscope/

[13] https://cividec.at/_C2_Broadband_Amplifier,2_GHz, 40_dB.html

[14] P. Moritz, E. Berdermann, K. Blasche, H. Stelzer, B. Voss, "Broadband electronics for CVD-diamond detectors", Diamond and Related Materials 10 pp 1765-1769, 2001.

[15] M. Schreck, J. Asmussen, S. Shikata, J.-C. Arnault, and N. Fujimori, "Large-area high-quality single crystal diamond," MRS Bull., vol. 39, no. 6 , pp. 504-510, Jun. 2014 